\title{
Air Microbial Contamination and Factors Affecting Its Occurrence in Certain Book Libraries In Egypt.
}

\author{
M.E. Osman a , A.A., Abdel Hameed b , H.Y., Ibrahim ${ }^{\text {b }}$, F, \\ Yousef $^{c}$, A.A., Abo Elnasr ${ }^{a}$ and Y. Saeed, ${ }^{b *}$. \\ ${ }^{a}$ Botany Department, Faculty of Science, Helwan University, \\ ${ }^{b}$ Air Pollution Department and ${ }^{c}$ Animal Reproduction and A.I. \\ Department, National Research Centre, Dokki, Giza, Egypt.
}

B ACTERIAL and fungal contamination in the air and settled dust were studied in old and new book libraries located in the National Research Center (NRC), Dokki, Giza, Egypt. The investigated libraries differ in age, design, size, ventilation type, and a number of occupants in relation to microclimatic parameters and particulate matter (PM) load. Airborne microorganisms were collected using an Andersen two stage impactor sampler. Indoor airborne bacteria and fungi ranged from $0-1060.4 \mathrm{CFU} / \mathrm{m}^{3}$ and $11.8-315.6 \mathrm{CFU} / \mathrm{m}^{3}$, respectively. Outdoor airborne bacteria and fungi ranged within $11.7-2514.7 \mathrm{CFU} / \mathrm{m}^{3}$ and 0 713.7 $\mathrm{CFU} / \mathrm{m}^{3}$, respectively. Bacteria and fungi associated surface settled. The dust ranged from $0.4-10 \times 10^{6} \mathrm{CFU} / \mathrm{gm}$ and $0-73 \times 10^{4}$ $\mathrm{CFU} / \mathrm{gm}$, respectively. Fine microbial fraction (particles $\leq 8 \mu \mathrm{m}$ in size) constituted $2-24.94 \%$ and $68.35-94.15 \%$ of the total airborne bacterial and fungal concentrations, respectively. Indoor/outdoor (I/O) ratios of airborne microorganisms were less than 1 at both libraries, indicating no indoor microbial sources. Gram positive cocci (14.3$47 \%$ ) and bacilli (52.9-85.7\%) were the dominant bacterial isolates in the air state, while bacilli represented $100 \%$ of the total isolates in the surface settled dust. Bacillus pseudomycoides and B. subtilis dominated indoors while B. subtilis and Staphylococcus outdoors. Aspergillus and Penicillium, were the common fungal species in both libraries under investigation. Many of the isolated fungal taxa had enzymatic activities (lipase, protease and cellulase), with A. flavus, Curvularia pallescens, Fusarium oxysporium, $P$. notatum and Trichoderma viride presented all enzymatic activities. Complex correlations and no-clear patterns were found between the airborne microorganisms and the environmental factors.

Keywords: Library, Microbial contamination, Particle Size, Identification, Enzymatic Activities, Microclimatic Factor. 
Libraries are institutions for preserving books and cultural heritage. Library collections undergo process of aging, alternation and deterioration by physical, chemical and biological agents (Gallo, 1993 and Maggi et al., 2000 \& Cappitelli et al., 2010). Organic matter (vegetable, animal and synthetic origins), relative humidity and dust are suitable conditions for microbial growth in libraries (Ciferri et al., 2003, Karbowska-Berent et al., 2011). Microorganisms may pose a danger to books and archival collections (Harkawy et al., 2011 and Tao et al., 2014) and to staff and visitors (Mesquita et al., 2009, Skóra et al., 2012).

Microbial aerosols in the library may exist as a result of infiltration of ambient air, human activity, air ventilation systems, presence of indoor reservoirs (ACGIH, 1999). Microorganisms are always attached dust particles (Alghamdi et al., 2014). Dust serves as a source of nutrients and forms a proper microenvironment on surfaces for microorganisms (Urzi and Realini, 1998). Water and suitable microclimatic conditions, e.g., temperature and relative humidity, are essential conditions for microbial growth indoors (Ritchkoff et al., 2000).

Fungi and bacteria may cause biological deterioration of materials, and there are many microbial taxa responsible for biodeterioration. Fungi cause great damage to cultural heritage because they possess high biodeteriogenic capacity of organic matter (Borrego and Perdomo, 2012). May et al. (1993) listed dematiaceae fungi among the major agents of biodeterioration of surfaces and dark spots. Urzi et al. (2001) concluded that once fungi settle on and colonize surfaces, they are responsible for a great variety of alternation like black paints, intergranular growth, sugaring and biopitting. Species of Trichoderma, Penicillium, Botrytis, Trichothecium, Phoma, Chaetomium, Aspergillus, Cladosporium, Stemphylium, Alternaria, Hormodendrum, Aureobasidium, Papularia, Bacillus, Cellulomonas, Cellfalciculata, Cellvibrio, Sporocytophaga and Streptomyces have celluolytic properties, Aureobasidium, Chaetomium, Cladosporium, Botrytis, Trichoderma, Verticillium, Mucor, Epicoccum, Gymnoascus and Actinomycetes have proteolytic properties, all the proteolytic fungi listed above and Paecilomyces have lipolytic properties (Borrego et al., 2010 and Berent et al., 2011).

Inhalation of microorganisms may cause health threats. Wiszniewska et al. (2009) identified allergy to fungi in $31 \%$ of staff working at the national museum in Warsaw. Mycotoxins producing fungi, e.g. Aspergillus, Penicillium, and Stachybotrytis have been isolated from museum, libraries and archive settings (Eduard, 2009 and Skóra et al., 2015).

The evaluation of microorganisms in air and surface dust is the first step to control library's environment and maintains deterioration. The present study aims to evaluate microbiological indoor air quality at two libraries and their relationships with microclimatic factors and particulate matter (PM). Moreover the potentiality of fungi, particularly biodegradable taxa, with cellulolytic, proteolytic and lipolytic enzymatic activities was evaluated. 


\section{Materials and Methods}

Sampling sites and strategy

The sampling was performed at two different libraries differ in age, size, design, height, location, ventilation, and number of books and users. The libraries are located at the main campus of the National Research Centre (NRC), Dokki, Giza governorate, Egypt. This is an urban area characterized by heavy traffic, parking, playgrounds, small workshops, hospitals, educational settings and hostels. A variety of vegetation is present in the area but there is no predominant ground cover.

The old is library of (National Center for Information and Documentation (NIDOC); affiliated to the Academy of Scientific Research \& Technology (ASRT) located at the $2^{\text {nd }}$ floor in the main building of the NRC. It was established in 1956 and characterized by fans and natural ventilation, with no air conditioning systems, and all windows are faced to the busy- traffic street (Fig. 1). On the other hand, the new library was built in 2009. It is located on the ground floor $\sim 75 \mathrm{~m}$ inside the main campus of the NRC away from the busy traffic street. It is fully air-conditioned library with low opening windows. The old library is a duplex floor, and it is $\sim 3$ times bigger in size than the new one; however the users and readers are higher in the new library than the old one. The two libraries had no historic indoor air quality issues.

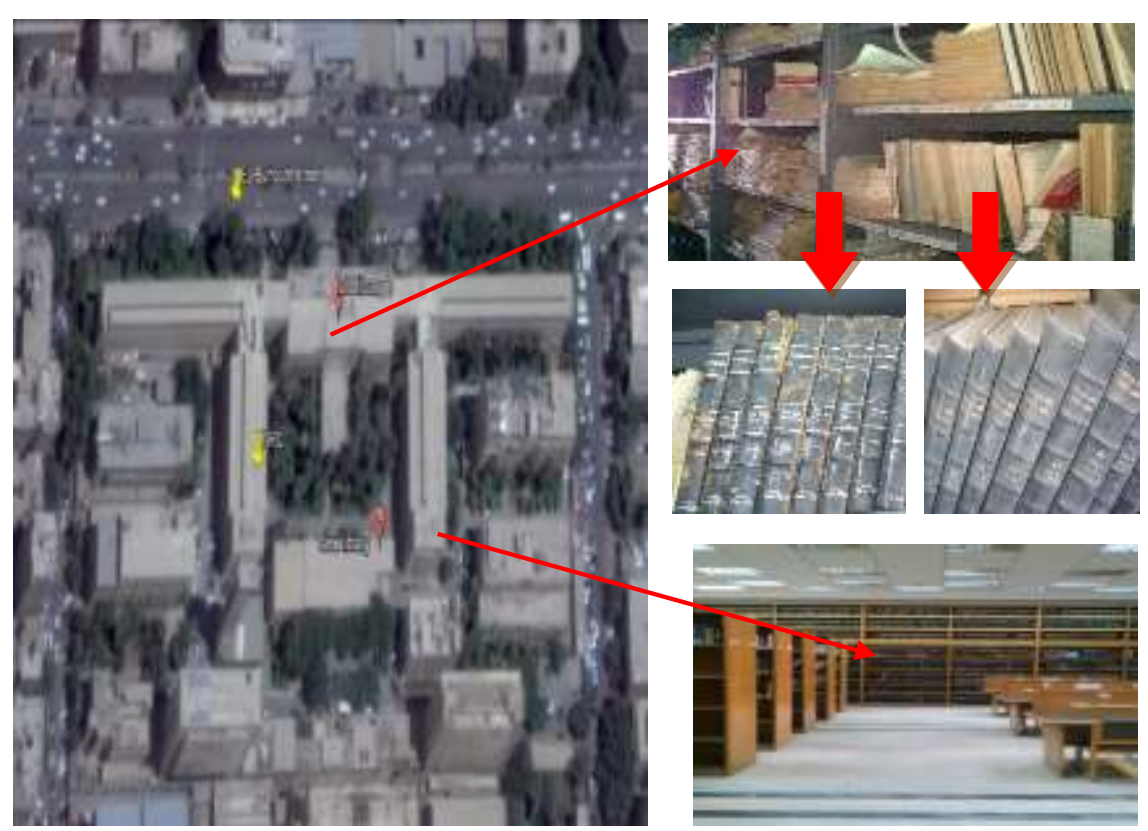

Fig. 1. Map showing locations of the both libraries at the NRC.

Egypt. J. Bot. 57, No.1(2017) 
Air samples were taken between January to December 2013 during the normal working days and human activities. Sampling was conducted between 9 am and $2 \mathrm{pm}$, during $10^{\text {th }}-15^{\text {th }}$ and $25^{\text {th }}-30^{\text {th }}$ days per month. Each library was scheduled for sampling twice per month, a total of 6 sampling events were conducted per season. The air samples were positioned at a height of $\sim 1.5 \mathrm{~m}$ above the floor level in the middle of the library. The comparison samples were taken 3-5 m outside the main front door of the new library and $50-75 \mathrm{~cm}$ of the window's ledge at the old library, facing to the main street.

A portable weather station (SATO, PC- 5000 TRH-II sampler) was used to determine the indoor and outdoor temperatures and relative humidity (microclimatic parameters) at the time of the microbial sample collection. Sampling was not take place during or within $48 \mathrm{hr}$ of a rain event. The ranges of libraries average temperatures were $17-29^{\circ} \mathrm{C}$ indoors and $16-32^{\circ} \mathrm{C}$ outdoors, while the ranges of libraries average relative humidity were $37-66 \%$ indoors and 28 $55 \%$ outdoors. Temperature and relative humidity records were slightly higher in the old library than the new one. No significant differences were found between the indoor temperature and relative humidity at both libraries. However significant difference $(\mathrm{t}=2.53, \mathrm{P} \leq 0.05)$ was observed between the outdoor relative humidity at both libraries.

\section{Microbiological sampling and analysis}

Airborne microorganisms including environmental bacteria, mesophilic bacteria and fungi were collected using an Andersen two-stage viable cascade impactor sampler (TE-10-160, Tisch Environmental Cleves, OH, USA). It separates particles into fine $(\leq 8 \mu \mathrm{m})$ and coarse $(\geq 8 \mu \mathrm{m})$ size ranges. Particle diameter determines where particles are likely to deposit in the respiratory tract (Thomas, 2013). Nutrient agar supplemented with cycloheximide and rosebengal streptomycin agar media were used to count bacteria and fungi, respectively (Mouli et al., 2005 and Sarica et al., 2005). The sampler was operated at a manufacturer recommended flow rate of $28.3 \mathrm{l} / \mathrm{min}$, for $5 \mathrm{~min}$. Because of the short sampling time two consecutive samples were taken $(1 \mathrm{hr}$ rest between sampling) during every sampling event (4 plates /indicator/location, a total of 24 plates/ sampling event).

The bacterial plates were incubated at $25^{\circ} \mathrm{C}$ for $48 \mathrm{hrs}$ for growing environmental bacteria and at $37{ }^{\circ} \mathrm{C}$ for $48 \mathrm{hrs}$ for growing mesophilic bacteria. Fungal plates were incubated at $28^{\circ} \mathrm{C}$ for 5-7 days and checked daily. Positive hole correction (Andersen, 1958) was applied to the raw colony forming unit (CFU) recorded on each plate and by using the CFU with sampling time and flow rate, the concentrations were calculated and expressed as the colony forming unit per cubic meter of the air $\left(\mathrm{CFU} / \mathrm{m}^{3}\right)$.

Surface dust accumulated over time on floor, books, shelves and furniture were collected, twice every month, using a brush and sterilized plastic bags, and microbiologically analyzed. The dust sample was thoroughly mixed and sieved using a sieve with pore diameter $\leq 45 \mu \mathrm{m}$ (Rumo shaker model 2200, U.K) to Egypt. J. Bot. 57, No.1(2017) 
remove particles $>45 \mu \mathrm{m}$. A weight of $0.05-0.1$ gm of dust $\leq 45 \mu \mathrm{m}$ was suspended in $25 \mathrm{ml}$ sterile distilled water and shaken well for 45-60 min. Aliquots $(0.5 \mathrm{ml})$ of the original sample and its serial dilutions $\left(10^{-2}\right)$ were spread plated, in duplicate, onto the surface of the nutrient agar and rose-bengal agar media and incubated as previously mentioned to count and identify bacteria and fungi associated dust, respectively. The concentrations of microorganisms associated dust were calculated and expressed as the colony forming unit per gram of dust (CFU/gm).

\section{Particulate matter (PM)}

Particulate matter (PM) samples were collected, indoors and outdoors at both libraries, using sterilized pre-weighted cellulose nitrate membrane filters $(0.45 \mu \mathrm{m}$ pore size and $25 \mathrm{~mm}$ diameter) equipped in open face filter holders and vacuum pumps calibrated to draw $16 \mathrm{l} / \mathrm{min}$. for $3 \mathrm{hrs}$. The filters were weighted (Sartorius TE2145, Germany), and along with sampling time and flow rate, the concentration was calculated and expressed as microgram per cubic meter of the $\operatorname{air}\left(\mu \mathrm{g} / \mathrm{m}^{3}\right)$.

\section{Identification of microorganisms}

Bacterial and fungal isolates that were isolated from the air and dust were identified. Bacterial isolates were identified by using BIOLOG 21124 (Cabot Blvd, Hayward, CA 94545, Water Pollution Dept., NRC, Egypt). Biolog's microbial identification system software (OmniLog ${ }^{\circledR}$ Data Collection) was used to identify the bacterium from its phenotypic pattern in the GEN III MicroPlate. On the other hand, fungal isolates were identified by the direct observation on the basis of micro-and macro- morphological features on Sabouraud dextrose agar, Czapek's dox agar and malt extract agar, reverse and surface coloration of colonies using various literature (Ellis, 1971; Barnett, 1972 and Raper \& Fennell, 1977; Singh et al., 1991 and Barnett \& Hunter, 1999; Hussein, 2002 and Pitt \& Hocking, 2009).

\section{Fungal enzymatic activity}

Cellulose, lipid and protein decomposing fungi were qualitatively screened using selective media. Cellulolytic activity was determined using cellulosecontaining agar medium $\left(0.03 \%\right.$ urea, $0.02 \% \mathrm{KH}_{2} \mathrm{PO}_{4}, 0.14 \%\left(\mathrm{NH}_{4}\right)_{2} \mathrm{SO}_{4}, 0.03 \%$ $\mathrm{MgSO}_{4} .7 \mathrm{H}_{2} \mathrm{O}, 0.5 \%$ cellulose, $0.1 \%$ peptone, $0.00001 \% \mathrm{FeSO}_{4} .7 \mathrm{H}_{2} \mathrm{O}, 0.00016 \%$ $\mathrm{MnSO}_{4} .7 \mathrm{H}_{2} \mathrm{O}, 0.00017 \% \mathrm{ZnSO}_{4} .7 \mathrm{H}_{2} \mathrm{O}, 0.0002 \% \mathrm{COCl}_{2}, 2 \%$ agar, pH 5), (Bland and Douglas, 1977). Mycelium discs, $5 \mathrm{~mm}$ diameter, from 7 days old culture fungal taxa were cut and placed onto the center of cellulose agar plates. The plates were incubated at $28 \pm 2{ }^{\circ} \mathrm{C}$ for $72 \mathrm{hrs}$, and further incubated at $50^{\circ} \mathrm{C}$ for 8 hrs (Osman et al., 2008). The plates were flooded with rose-bengal $(0.005 \%)$. The appearance of clear zone around the colony discs indicates cellulolytic activity (Bland and Douglas, 1977).

Lipase activity was screened using phenol red agar plates $(0.01 \%$ phenol red, $1 \%$ olive oil, $0.1 \% \mathrm{CaCl}_{2}$ and $2 \%$ agar, $\mathrm{pH} 7.4$ ), (Singh et al., 2006). Mycelium discs, $5 \mathrm{~mm}$ diameter, from 7 days old culture fungal taxa were cut and placed 
onto the center of agar plates. The plates were incubated at $37{ }^{\circ} \mathrm{C}$ for 4 days. The appearance of yellow color, due to release of fatty acid, indicates lipase enzymatic activity.

Protease activity was determined using Gelatin-agar medium plates (1\% gelatin, 2\% agar and $0.02 \% \mathrm{M}$ phosphate buffer, pH. 7.5), (Ammar et al., 1991). Mycelium discs, $5 \mathrm{~mm}$ diameter, from 7 days old culture fungal taxa were cut and placed onto the center of agar plates and incubated at $25^{\circ} \mathrm{C}$ for 4 days. The plates were flooded with $10 \mathrm{ml}$ acidic mercuric chloride solution (Cowan, 1974), and the appearance of clear zone around the colonies (discs) indicates protease enzymatic activity.

\section{Medical effects}

An interview was performed among all employees working at both libraries. The questionnaire contained general questions on the frequency of the symptoms for 3 days per week in the previous 4 weeks before survey, or which appear every working day and got better when the employee went away from the work (Crandell et al., 1996). The questionnaire contained the following general symptoms:

- Shortness of breath, cough and wheezing;

- Dry eye, watering eyes, irritation of the eyes, and allergic conjunctivitis;

- $\quad$ Sneezing, stuffy nose, coryza and throat irritation;

- Headache, fatigue, drowsiness, dizziness and nausea and

- Dermatitis

\section{Statistical analysis}

The Mann Whitney $U$ test was used to ascertain the significance of differences between microclimatic conditions at both libraries as well as airborne microorganisms inside and outside the libraries. Spearman's rank correlation test was used to determine the relationships between airborne microbial concentrations with both microclimatic conditions and particulate matter (PM). A probability of less or equal to $\mathrm{P} \leq 0.05$ was considered significant.

\section{Results}

\section{Microbiological air quality}

Table 1 and Fig.2. show the mean total airborne microbial concentrations at the two libraries. Environmental bacteria ranged from $0-1060.4 \mathrm{CFU} / \mathrm{m}^{3}$ indoors and $11.77-2514.71 \mathrm{CFU} / \mathrm{m}^{3}$ outdoors, higher concentrations shifted toward the old library. The maximum mean concentrations of environmental bacteria were 296.2 $\mathrm{CFU} / \mathrm{m}^{3}$ indoor and $1455.5 \mathrm{CFU} / \mathrm{m}^{3}$ outdoor. Mesophilic bacteria ranged within $0-405 \mathrm{CFU} / \mathrm{m}^{3}$ indoors and $11.77-2157.82 \mathrm{CFU} / \mathrm{m}^{3}$ outdoors, with higher concentrations outdoors.

Fungal concentrations ranged between 11.77 - $315.66 \mathrm{CFU} / \mathrm{m}^{3}$ indoors and 0$713.77 \mathrm{CFU} / \mathrm{m}^{3}$ outdoors. Generally, indoor and outdoor airborne microbial Egypt. J. Bot. 57, No.1(2017) 
concentrations were higher at the old library than the new one. No significant differences were found between airborne microbial concentrations indoors and outdoors at both libraries, except mesophilic bacteria that showed a significant difference in the outdoor and indoor concentrations $(\mathrm{P}=0.0286)$ at the old library, higher concentrations shifted toward outdoor.

TABLE 1. The range and mean concentrations $\left(\mathrm{CFU} / \mathrm{m}^{3}\right)$ of total airborne microorganisms at two libraries at the main campus of the National Research Centre (NRC), Dokki, Giza.

\begin{tabular}{|c|c|c|c|c|}
\hline \multirow{2}{*}{ Microbial type } & \multicolumn{3}{|c|}{ CFU/m } \\
\cline { 2 - 5 } & \multicolumn{2}{|c|}{ Old Library } & \multicolumn{2}{c|}{ New Library } \\
\cline { 2 - 5 } & Indoor & Outdoor & Indoor & Outdoor \\
\hline $\begin{array}{c}\text { Environmental } \\
\text { bacteria }\end{array}$ & $0.0-1060.4$ & $35.32-1107.18$ & $0.0-353.34$ & $11.77-2514.71$ \\
& $(167.61 \pm 296.2)$ & $(961.41 \pm 1455.5)$ & $(101.41 \pm 106.7)$ & $(738.72 \pm 644.9)$ \\
\hline Mesophilic & $0.0-107.17$ & $23.55-2157.82$ & $0-405.17$ & $11.77-1571.25$ \\
bacteria & $(32.45 \pm 28.9)$ & $(392.362 \pm 554.7)$ & $(67.57 \pm 99.3)$ & $(312.34 \pm 243)$ \\
\hline Fungi & $11.77-315.66$ & $0.0-713.77$ & $11.77-252.05$ & $47.11-374.55$ \\
& $(88.03 \pm 83.3)$ & $(185.35 \pm 185.4)$ & $(88.47 \pm 67.7)$ & $(188.89 \pm 136.6)$ \\
\hline
\end{tabular}

Range, (Mean \pm SD)

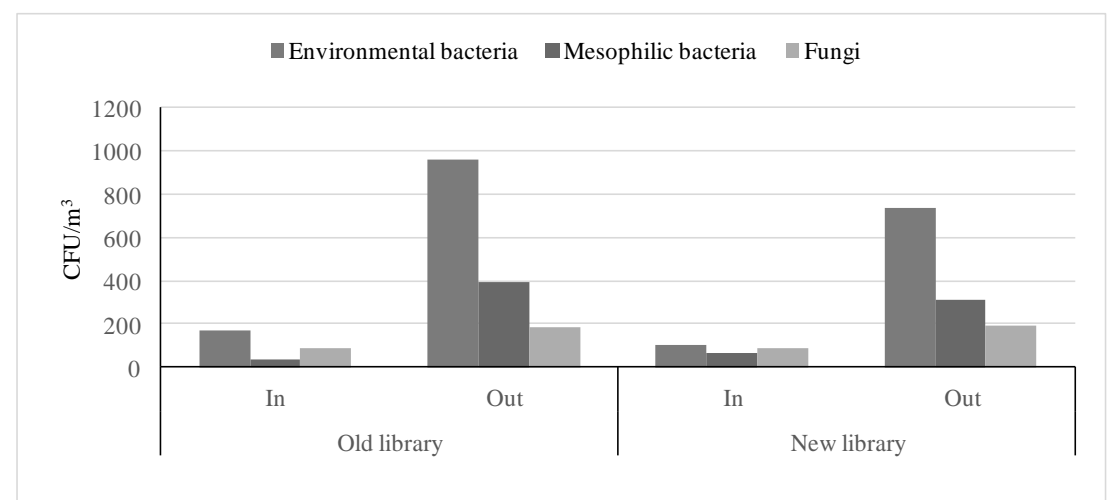

Fig. 2. Mean concentrations of total airborne microorganisms registered at the both libraries.

\section{Microbial size fraction}

The diameter of a microbial particle will impact how deep the particle may penetrate into the lungs. The mean concentrations of fine $(\leq 8 \mu \mathrm{m})$ and coarse $(\geq$ $8 \mu \mathrm{m})$ culturable airborne microbial concentrations are shown in Table 2 . The fine size fraction of environmental bacteria ranged from $0-1036.51 \mathrm{CFU} / \mathrm{m}^{3}$ indoors and $0-4030.62 \mathrm{CFU} / \mathrm{m}^{3}$ outdoors, while mesophilic bacterial concentrations ranged within $0-35.33 \mathrm{CFU} / \mathrm{m}^{3}$ indoors and $0-254.41 \mathrm{CFU} / \mathrm{m}^{3}$ outdoors. In contrast, fine size fraction of fungal concentrations ranged within 0 - 315.6 $\mathrm{CFU} / \mathrm{m}^{3}$ indoors and $0-508.83 \mathrm{CFU} / \mathrm{m}^{3}$ outdoors. 
TABLE 2. The range and mean concentrations of coarse $(\geq 8 \mu \mathrm{m})$ and fine $(8 \leq \mu \mathrm{m})$ culturable airborne microorganisms at two libraries at the main campus of the National Research Centre (NRC), Dokki, Giza.

\begin{tabular}{|c|c|c|c|c|c|c|}
\hline \multirow{4}{*}{ Location } & \multicolumn{6}{|c|}{$\mathrm{CFU} / \mathrm{m}^{3}$} \\
\hline & \multicolumn{4}{|c|}{ Bacteria } & \multirow{2}{*}{\multicolumn{2}{|c|}{ Fungi }} \\
\hline & \multicolumn{2}{|c|}{ Environmental } & \multicolumn{2}{|c|}{ Mesophilic } & & \\
\hline & $\geq 8 \mu \mathrm{m}$ & $\leq 8 \mu \mathrm{m}$ & $\geq 8 \mu \mathrm{m}$ & $\leq 8 \mu \mathrm{m}$ & $\geq 8 \mu \mathrm{m}$ & $\leq 8 \mu \mathrm{m}$ \\
\hline $\begin{array}{l}\text { Old } \\
\text { Indoor }\end{array}$ & $\begin{array}{c}0.0-1036.51 \\
(149.2 \pm 294.9)\end{array}$ & $\begin{array}{c}0.0-58.89 \\
(18.4 \pm 17.6)\end{array}$ & $\begin{array}{c}0.0-83.62 \\
(24.4 \pm 23.2)\end{array}$ & $\begin{array}{c}0.0-35.33 \\
(8.09 \pm 9.9)\end{array}$ & $\begin{array}{c}0.0-23.55 \\
(5.15 \pm 7.2)\end{array}$ & $\begin{array}{c}11.77-315.66 \\
(82.9 \pm 81.7)\end{array}$ \\
\hline Outdoor & $\begin{array}{c}23.6-4030.62 \\
(858.2 \pm 1241.9)\end{array}$ & $\begin{array}{c}0.0-849.23 \\
(103.2 \pm 225.3)\end{array}$ & $\begin{array}{c}23.55-1903.4 \\
(356.4 \pm 496.18)\end{array}$ & $\begin{array}{c}0.0-254.41 \\
(35.9 \pm 68.6)\end{array}$ & $\begin{array}{c}0.0-204.94 \\
(53.4 \pm 48.3)\end{array}$ & $\begin{array}{c}0.0-508.83 \\
(131.1 \pm 144.5)\end{array}$ \\
\hline $\begin{array}{l}\text { New } \\
\text { Indoor }\end{array}$ & $\begin{array}{c}0.0-329.79 \\
(89.6 \pm 101.9)\end{array}$ & $\begin{array}{c}0.0-58.33 \\
(11.8 \pm 15.6)\end{array}$ & $\begin{array}{c}0.0-393.4 \\
(60.2 \pm 96.6)\end{array}$ & $\begin{array}{l}0.0-23.55 \\
(7.4 \pm 7.0)\end{array}$ & $\begin{array}{c}0.0-35.33 \\
(15.5 \pm 14.3)\end{array}$ & $\begin{array}{l}0.0-216.72 \\
(73 \pm 64.2)\end{array}$ \\
\hline Outdoor & $\begin{array}{c}0.0-1765.6 \\
(719.5 \pm 623.8)\end{array}$ & $\begin{array}{c}0.0-107.2 \\
(19.2 \pm 28.7)\end{array}$ & $\begin{array}{c}0.0-1418.1 \\
(298.4 \pm 377.9)\end{array}$ & $\begin{array}{c}0.0-153.12 \\
(13.9 \pm 36.4)\end{array}$ & $\begin{array}{c}35.3-130.74 \\
(59.8 \pm 24.8)\end{array}$ & $\begin{array}{c}0.0-405.18 \\
(129.1 \pm 128.4)\end{array}$ \\
\hline
\end{tabular}

Range, (Mean \pm SD).

Fine fraction of environmental bacteria represented small percentages in indoors $(11-11.6 \%)$ and outdoors (2.6- 10.74\%), while mesophilic bacteria represented relatively higher percentages indoors $(10.87-24.94 \%)$ and outdoors $(4.48-9.16 \%)$. Fine fraction of fungi constituted the majority of size ranges $\sim 82.54-94.15 \%$ indoors and 68.35- $71.16 \%$ outdoors. Significant differences were found between the concentrations of coarse fractions of mesophilic bacteria $(P=0.0286)$ and fungi $(\mathrm{P}=$ 0.0143 ) registered in the outdoor and indoor at the old library and the new library, respectively, higher concentrations shifted toward outdoors.

\section{Indoor / Outdoor $(\mathrm{I} / \mathrm{O})$ ratio}

The I/O ratios of airborne microbial concentrations are illustrated in Fig. 3. I/O ratios were always less than 1 at both libraries. The I/O ratios for environmental bacteria, mesophilic bacteria and fungi reached $0.24,0.08$ and 0.47 , respectively at the old library, and they were $0.13,0.21$ and 0.46 for the corresponding microbial parameters at the new library. I/O ratios of environmental bacteria were relatively higher at the old library while mesophilic bacteria at the new library (Fig. 3).

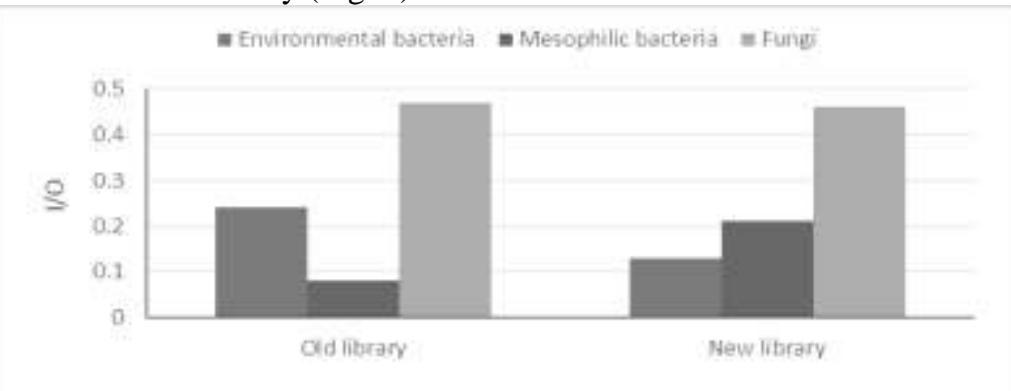

Fig. 3. I/O ratios of total airborne microorganisms at the both libraries

Egypt. J. Bot. 57, No.1(2017) 
Microorganisms associated surface dust

Table 3 shows range and mean concentrations of bacteria and fungi associated surface settled dust at the two libraries. Concentrations of bacteria associated dust exceeded fungi; however the environmental bacteria were higher than mesophilic ones. Environmental bacteria associated settled dust ranged from $1.2 \times 10^{5}-7.9 \times 10^{6} \mathrm{CFU} / \mathrm{gm}$ indoors and $6 \times 10^{4}-10 \times 10^{6} \mathrm{CFU} / \mathrm{gm}$ outdoors, while mesophilic bacteria ranged within $4 \times 10^{4}-5.68 \times 10^{6} \mathrm{CFU} / \mathrm{gm}$ indoors and $6 \times 10^{4}-3.3 \times 10^{6} \mathrm{CFU} / \mathrm{gm}$ outdoors. Fungal concentrations were found in the range of $0-6.3 \times 10^{5} \mathrm{CFU} / \mathrm{gm}$ indoors and $1 \times 10^{4}-7.5 \times 10^{5} \mathrm{CFU} / \mathrm{gm}$ outdoors. At the old library, fungi associated dust concentrations significantly differed $(\mathrm{P}=0.0143)$ indoor and outdoor. Moreover indoor fungal concentrations $(\mathrm{P}=$ 0.0143 ) significantly differed at both libraries, higher concentrations shifted towards the new library.

TABLE 3. The range and mean concentrations of microorganisms associated settled dust $(\leq 45 \mu \mathrm{m})$ at two libraries at the main campus of the National Research Centre (NRC), Dokki, Giza.

\begin{tabular}{|c|c|c|c|c|c|c|}
\hline \multirow{4}{*}{ Location } & \multicolumn{4}{|c|}{ CFU/gm $\times 10^{5}$} & \multirow{3}{*}{\multicolumn{2}{|c|}{$\frac{\text { CFU/gm } \times 10^{4}}{\text { Fungi }}$}} \\
\hline & \multicolumn{4}{|c|}{ bacteria } & & \\
\hline & \multicolumn{2}{|c|}{ Environmental } & \multicolumn{2}{|c|}{ Mesophilic } & & \\
\hline & Range & Mean \pm SD & Range & Mean \pm SD & Range & Mean \pm SD \\
\hline Old Indoor & $1.2-79.0$ & $12.08 \pm 18.55$ & $0.4-48.8$ & $10.72 \pm 13.91$ & $0.0-7.0$ & $2.4 \pm 1.5$ \\
\hline Outdoor & $0.6-100$ & $19.93 \pm 26.11$ & $0.6-30.0$ & $6.17 \pm 8.16$ & $1.0-75.0$ & $12.1 \pm 14.7$ \\
\hline NewIndoor & $3.2-47.2$ & $19.55 \pm 13.69$ & $1.8-56.8$ & $9.51 \pm 11.16$ & $2.0-63.0$ & $11.0 \pm 12.1$ \\
\hline Outdoor & $10.0-60.6$ & $31.52 \pm 19.08$ & $3.2-33.0$ & $14.45 \pm 8.27$ & $1.0-50.0$ & $10.0 \pm 11.1$ \\
\hline
\end{tabular}

\section{Particulate matter}

The mean concentrations of PM at both libraries are graphically illustrated in Fig. 4. PM concentrations ranged between $203.52-395.16 \mu \mathrm{g} / \mathrm{m}^{3}$, with the greatest mean values of $395.16 \mu \mathrm{g} / \mathrm{m}^{3}$ indoors and $306 \mu \mathrm{g} / \mathrm{m}^{3}$ outdoors. PM concentrations were higher at the old library with I/O ratio exceeded 1 . Significant difference was found between the outdoor PM concentrations at the new and old libraries $(\mathrm{P}=0.0143)$, higher concentrations shifted toward the old library.

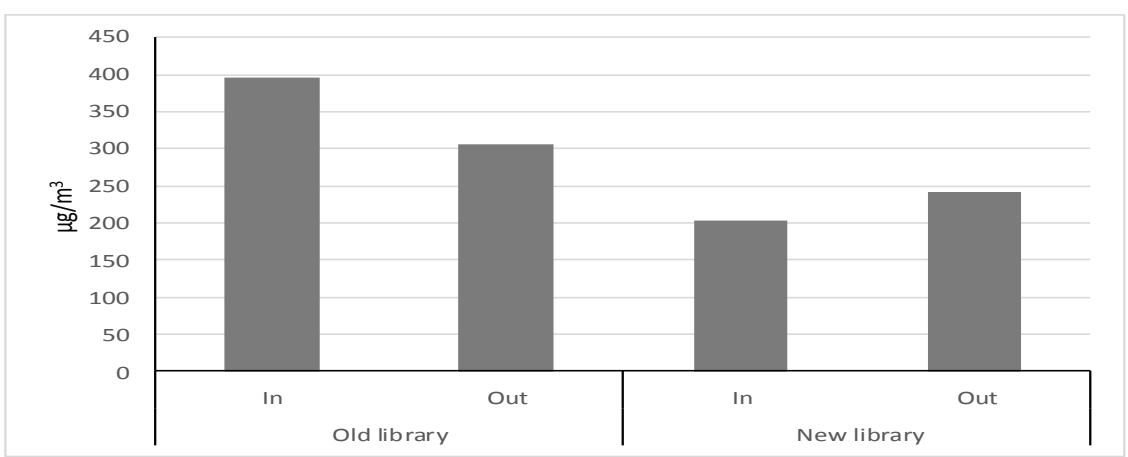

Fig. 4. The mean concentrations of PM indoors and outdoors of the both libraries. 
Influence of environmental factors on microbial survivability

Spearman's rank correlations between airborne microorganisms with temperature, relative humidity and PM are shown in Table 4. Complex correlations were found between airborne microorganisms and environmental factors. Generally, temperature negatively affected bacterial survivability at both libraries. Significant negative correlations $(\mathrm{P} \leq 0.05)$ were found between temperature and airborne bacteria, both environmental and mesophilic bacteria, at the old library. Significant negative correlations were found between temperature and fungi in outdoor the old library $(\mathrm{r}=-0.67)$ and indoor the new library $(\mathrm{r}=-$ 0.61), (Table 4). Relative humidity (RH\%) showed significant positive correlations with indoor and outdoor environmental bacteria at the old library. However, significant positive correlations were found between relative humidity and mesophilic bacteria outdoor the old library $(\mathrm{r}=0.77)$ and indoor the new library $(\mathrm{r}=0.51)$. In contrast, negative and positive correlations were found between RH\% with indoor and outdoor fungi at the old and new libraries, respectively.

TABLE 4. Spearman's rank correlation coefficients between airborne microorganisms with microclimatic parameters and particulate matter (PM).

\begin{tabular}{|l|c|c|c|c|c|c|c|c|c|}
\hline \multirow{3}{*}{ Location } & \multicolumn{3}{|c|}{ T $^{\circ} \mathbf{C}$} & \multicolumn{3}{c|}{ RH\% } & \multicolumn{3}{c|}{ PM } \\
\cline { 2 - 6 } & \multicolumn{2}{|c|}{ Bacteria } & \multirow{2}{*}{ Fungi } & \multicolumn{2}{c|}{ Bacteria } & \multirow{2}{*}{ Fungi } & \multicolumn{2}{c|}{ Bacteria } & \multirow{2}{*}{ Fungi } \\
\cline { 2 - 7 } & Env. & Meso. & & Env. & Meso. & & Env. & Meso. & \\
\hline \multirow{2}{*}{ Old Indoor } & $-0.61^{*}$ & $-0.67^{*}$ & 0.22 & $0.76^{*}$ & 0.12 & -0.32 & 0.33 & 0.15 & $-0.62^{*}$ \\
\hline Outdoor & $-0.82^{*}$ & $-0.75^{*}$ & $-0.67^{*}$ & $0.66^{*}$ & $0.77^{*}$ & $-0.58^{*}$ & 0.2 & $0.59^{*}$ & 0.052 \\
\hline New Indoor & -0.42 & -0.29 & $-0.61^{*}$ & 0.42 & $0.51^{*}$ & $0.61^{*}$ & $-0.8^{*}$ & $-0.77^{*}$ & $-0.44^{*}$ \\
\hline Outdoor & $-0.48^{*}$ & -0.13 & 0.1 & 0.40 & -0.06 & 0.16 & -0.26 & 0.26 & 0.20 \\
\hline
\end{tabular}

Env: Environmental bacteria; Meso: Mesophilic bacteria;* significant $(\mathrm{p} \leq 0.05)$.

PM showed positive correlations with airborne bacteria at the old library and negative correlations with indoor and outdoor airborne bacteria at the new library, except with outdoor airborne mesophilic bacteria (positively correlated with PM). Positive and negative correlations were found between PM with fungi outdoors and indoors, respectively (Table 4).

\section{Microbial biodiversity}

Table 5 shows percentages of bacterial taxa isolated from the air state and surface settled dust. No Gram negative bacteria were isolated, however Gram positive cocci and bacilli constituted 14.28 - 47.06\% and 52.94-85.72\%, respectively of the total bacterial isolates. Bacillus pseudomycoides and $B$. subtilis were frequently found indoors while B. subtilis and Staphylococcus outdoors. Alloiococcus otitis and Macrococcus caseolyticus were only found in the air state at the new library. Bacillus was found to be the only genus associated surface settled dust, and represented by: B. atrophaeus, B. pumilus, B. subtilis and Paenibacillus glucanolyticus.

Egypt. J. Bot. 57, No.1(2017) 
TABLE 5. Bacterial taxa isolated from the air state and settled dust at two libraries at the main campus of the National Research Centre (NRC), Dokki, Giza.

\begin{tabular}{|c|c|c|c|c|c|c|c|c|c|}
\hline \multirow{4}{*}{ Species } & \multirow{4}{*}{ Medium } & \multicolumn{8}{|c|}{ Bacteria \% } \\
\hline & & \multicolumn{4}{|c|}{ Environmental } & \multicolumn{4}{|c|}{ Mesophilic } \\
\hline & & \multicolumn{2}{|c|}{ Old Library } & \multicolumn{2}{|c|}{ New Library } & \multicolumn{2}{|c|}{ Old Library } & \multicolumn{2}{|c|}{ New Library } \\
\hline & & In & Out & In & Out & In & Out & In & Out \\
\hline Alloiococcus otitis & $\begin{array}{c}\text { Air } \\
\text { Dust }\end{array}$ & $\begin{array}{l}0 \\
0\end{array}$ & $\begin{array}{l}0 \\
0\end{array}$ & $\begin{array}{c}9.52 \\
0\end{array}$ & $\begin{array}{l}0 \\
0\end{array}$ & $\begin{array}{l}0 \\
0\end{array}$ & $\begin{array}{l}0 \\
0\end{array}$ & $\begin{array}{l}0 \\
0\end{array}$ & $\begin{array}{l}5 \\
0\end{array}$ \\
\hline Bacillus atrophaeus & $\begin{array}{l}\text { Air } \\
\text { Dust }\end{array}$ & $\begin{array}{c}0 \\
50\end{array}$ & $\begin{array}{c}0 \\
12.5\end{array}$ & $\begin{array}{c}4.76 \\
27.28\end{array}$ & $\begin{array}{c}5 \\
50\end{array}$ & $\begin{array}{c}15 \\
44.45\end{array}$ & $\begin{array}{l}15.38 \\
22.22\end{array}$ & $\begin{array}{l}14.28 \\
27.28\end{array}$ & $\begin{array}{c}20 \\
44.44\end{array}$ \\
\hline Bacillus licheniformis & $\begin{array}{l}\text { Air } \\
\text { Dust }\end{array}$ & $\begin{array}{c}50 \\
0\end{array}$ & $\begin{array}{l}0 \\
0\end{array}$ & $\begin{array}{c}9.52 \\
0\end{array}$ & $\begin{array}{l}5 \\
0\end{array}$ & $\begin{array}{l}5 \\
0\end{array}$ & $\begin{array}{l}0 \\
0\end{array}$ & $\begin{array}{l}0 \\
0\end{array}$ & $\begin{array}{l}0 \\
0\end{array}$ \\
\hline $\begin{array}{l}\text { Bacillus } \\
\text { pseudomycoides }\end{array}$ & $\begin{array}{l}\text { Air } \\
\text { Dust }\end{array}$ & $\begin{array}{c}10 \\
0\end{array}$ & $\begin{array}{l}0 \\
0\end{array}$ & $\begin{array}{c}4.76 \\
0\end{array}$ & $\begin{array}{c}15 \\
0\end{array}$ & $\begin{array}{c}15 \\
0\end{array}$ & $\begin{array}{c}7.69 \\
0\end{array}$ & $\begin{array}{c}14.28 \\
0\end{array}$ & $\begin{array}{c}10 \\
0\end{array}$ \\
\hline Bacillus pumilus & $\begin{array}{l}\text { Air } \\
\text { Dust }\end{array}$ & $\begin{array}{c}0 \\
37.5\end{array}$ & $\begin{array}{c}11.76 \\
25\end{array}$ & $\begin{array}{c}0 \\
36.36\end{array}$ & $\begin{array}{c}5 \\
25\end{array}$ & $\begin{array}{c}0 \\
55.55\end{array}$ & $\begin{array}{c}0 \\
44.45\end{array}$ & $\begin{array}{l}14.28 \\
54.54\end{array}$ & $\begin{array}{c}5 \\
11.12\end{array}$ \\
\hline Bacillus subtilis & $\begin{array}{l}\text { Air } \\
\text { Dust }\end{array}$ & $\begin{array}{c}20 \\
12.5\end{array}$ & $\begin{array}{c}41.18 \\
50\end{array}$ & $\begin{array}{l}19.04 \\
36.36\end{array}$ & $\begin{array}{l}15 \\
25\end{array}$ & $\begin{array}{c}30 \\
0\end{array}$ & $\begin{array}{l}26.92 \\
33.33\end{array}$ & $\begin{array}{c}28.6 \\
18.18\end{array}$ & $\begin{array}{c}15 \\
44.44\end{array}$ \\
\hline $\begin{array}{l}\text { Macrococcus } \\
\text { caseolyticus }\end{array}$ & $\begin{array}{l}\text { Air } \\
\text { Dust }\end{array}$ & $\begin{array}{l}0 \\
0\end{array}$ & $\begin{array}{l}0 \\
0\end{array}$ & $\begin{array}{c}4.76 \\
0\end{array}$ & $\begin{array}{l}0 \\
0\end{array}$ & $\begin{array}{l}0 \\
0\end{array}$ & $\begin{array}{l}0 \\
0\end{array}$ & $\begin{array}{l}0 \\
0\end{array}$ & $\begin{array}{l}0 \\
0\end{array}$ \\
\hline $\begin{array}{l}\text { Paenibacillus } \\
\text { amylolyticus }\end{array}$ & $\begin{array}{c}\text { Air } \\
\text { Dust }\end{array}$ & $\begin{array}{l}0 \\
0\end{array}$ & $\begin{array}{l}0 \\
0\end{array}$ & $\begin{array}{c}14.28 \\
0\end{array}$ & $\begin{array}{c}25 \\
0\end{array}$ & $\begin{array}{l}0 \\
0\end{array}$ & $\begin{array}{c}3.84 \\
0\end{array}$ & $\begin{array}{c}7.14 \\
0\end{array}$ & $\begin{array}{c}30 \\
0\end{array}$ \\
\hline $\begin{array}{l}\text { Paenibacillus } \\
\text { glucanolyticus }\end{array}$ & $\begin{array}{c}\text { Air } \\
\text { Dust }\end{array}$ & $\begin{array}{l}0 \\
0\end{array}$ & $\begin{array}{c}0 \\
12.5\end{array}$ & $\begin{array}{c}9.52 \\
0\end{array}$ & $\begin{array}{l}0 \\
0\end{array}$ & $\begin{array}{l}5 \\
0\end{array}$ & $\begin{array}{l}0 \\
0\end{array}$ & $\begin{array}{c}7.14 \\
0\end{array}$ & $\begin{array}{l}0 \\
0\end{array}$ \\
\hline Rothia nasimurium & $\begin{array}{l}\text { Air } \\
\text { Dust }\end{array}$ & $\begin{array}{l}0 \\
0\end{array}$ & $\begin{array}{c}11.76 \\
0\end{array}$ & $\begin{array}{c}4.76 \\
0\end{array}$ & $\begin{array}{l}0 \\
0\end{array}$ & $\begin{array}{c}10 \\
0\end{array}$ & $\begin{array}{c}23.1 \\
0\end{array}$ & $\begin{array}{c}14.28 \\
0\end{array}$ & $\begin{array}{c}10 \\
0\end{array}$ \\
\hline $\begin{array}{l}\text { Staphylococcus } \\
\text { aureus }\end{array}$ & $\begin{array}{l}\text { Air } \\
\text { Dust }\end{array}$ & $\begin{array}{c}10 \\
0\end{array}$ & $\begin{array}{c}17.65 \\
0\end{array}$ & $\begin{array}{c}19.08 \\
0\end{array}$ & $\begin{array}{c}15 \\
0\end{array}$ & $\begin{array}{l}5 \\
0\end{array}$ & $\begin{array}{c}15.38 \\
0\end{array}$ & $\begin{array}{l}0 \\
0\end{array}$ & $\begin{array}{l}0 \\
0\end{array}$ \\
\hline $\begin{array}{l}\text { Staphylococcus } \\
\text { species }\end{array}$ & $\begin{array}{l}\text { Air } \\
\text { Dust }\end{array}$ & $\begin{array}{c}10 \\
0\end{array}$ & $\begin{array}{c}17.65 \\
0\end{array}$ & $\begin{array}{l}0 \\
0\end{array}$ & $\begin{array}{c}15 \\
0\end{array}$ & $\begin{array}{c}15 \\
0\end{array}$ & $\begin{array}{c}7.69 \\
0\end{array}$ & $\begin{array}{l}0 \\
0\end{array}$ & $\begin{array}{l}5 \\
0\end{array}$ \\
\hline
\end{tabular}


The biodiversity of fungal taxa isolated from air state and surface settled dust is illustrated graphically in Fig. 5 a \&b. A total of 15 fungal taxa were identified as Aspergillus, Penicillium and Cladosporium were the predominant types at both libraries. A relatively different fungal content was found between the air state and surface settled dust. Monilia and Mucor species were only isolated from the air state at the old library. Epicoccum and Nigrospora species were only isolated from the surface settled dust at the old library while Trichoderma viride at the new library.
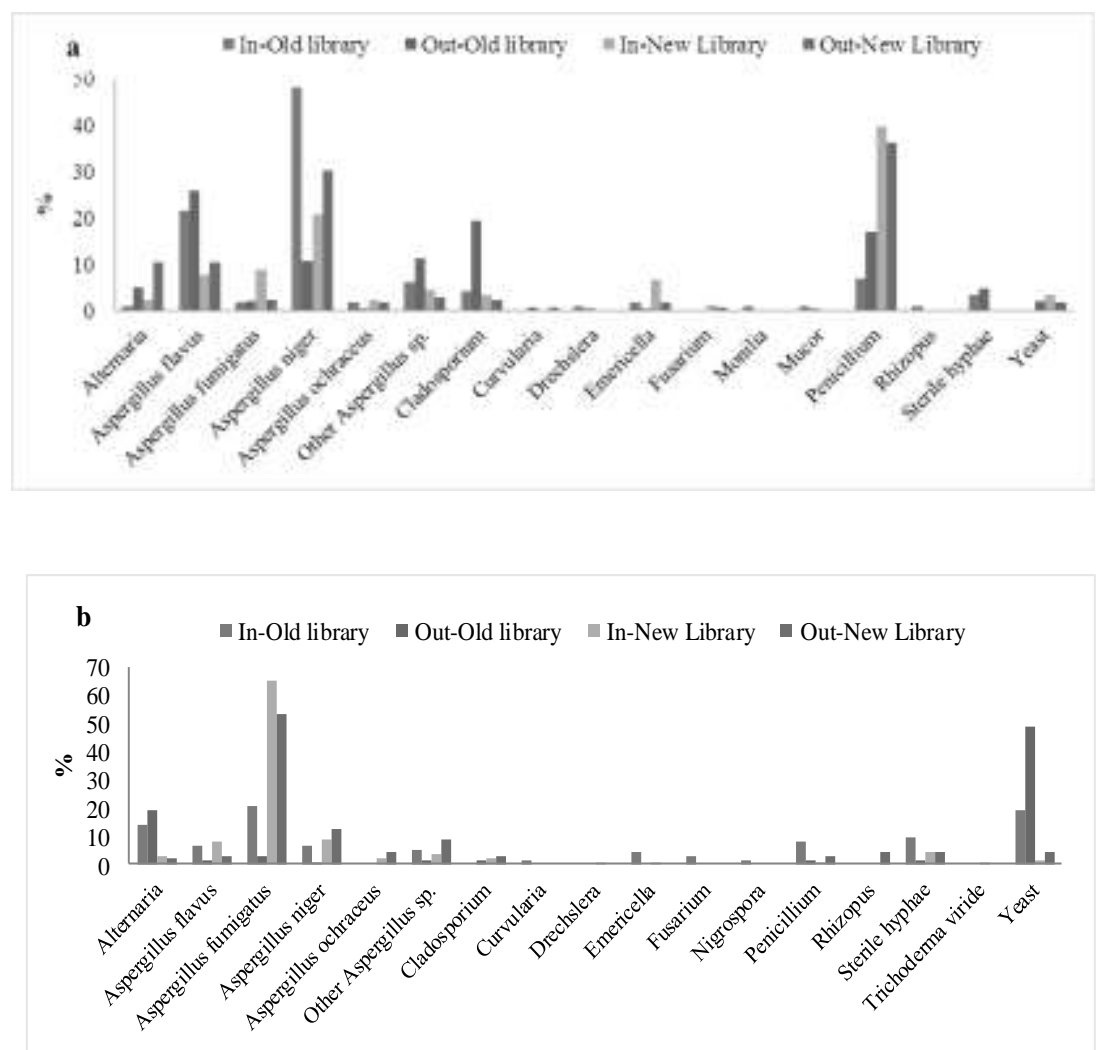

Fig. 5. Percentages of the identified fungal taxa isolated from air state (a) and settled dust (b) at two libraries at the main campus of the National Research Centre (NRC), Dokki, Giza.

\section{Enzymatic activities}

A total of 22 fungal isolates were screened for proteolytic, lipolytic and cellulolytic enzyme activities (Table 6). Many of the isolated fungal taxa had more than one of the enzymatic properties/activities. The majority of fungal taxa had cellulolytic (16 taxa) and lipolytic (15 taxa) properties and only 10 fungal

Egypt. J. Bot. 57, No.1(2017) 
taxa had proteolytic properties. Five fungal taxa namely: Aspergillus flavus, Curvularia pallescens, Fusarium oxysporium, Penicillium notatum and Trichoderma viride had proteolytic, lipolytic and cellulolytic properties.

\section{Medical effects}

Table 7 shows the health complaints among employees at both libraries. The employees at the new library had lower complaints for most of symptoms than those at the old library. Symptoms of the central nervous system including headache and fatigue were the common complaints among employees at both libraries. Higher portions of employees at the old library reported wheezing, shortness of breath, irritation of the eyes, cough, drowsiness, dizziness, dermatitis and throat irritation compared to those reported among employees at the new library.

TABLE 6. Qualitative detection of cellulases, lipases and proteases in the isolated fungal taxa.

\begin{tabular}{|c|c|c|c|}
\hline \multirow{2}{*}{ Species } & \multicolumn{3}{|c|}{ Enzyme } \\
\hline & Cellulase & Lipase & Protease \\
\hline Alternaria alternata & $\sqrt{ }$ & $\sqrt{ }$ & $N D$ \\
\hline Aspergillus flavus $^{I}$ & $\sqrt{ }$ & $\sqrt{ }$ & $N D$ \\
\hline A. flavus $^{2}$ & $\sqrt{ }$ & $\sqrt{ }$ & $\sqrt{ }$ \\
\hline A. fumigatus & $N D$ & $\sqrt{ }$ & $N D$ \\
\hline A. nidulans & $\sqrt{ }$ & $N D$ & $\sqrt{ }$ \\
\hline A. niger & $N D$ & $\sqrt{ }$ & $N D$ \\
\hline A. ochraceus & $\sqrt{ }$ & $N D$ & $\sqrt{ }$ \\
\hline Cladosporium cladosporioides & $\sqrt{ }$ & $N D$ & $N D$ \\
\hline Curvularia lunata & $\sqrt{ }$ & $N D$ & $\sqrt{ }$ \\
\hline Curvularia pallescens & $\sqrt{ }$ & $\sqrt{ }$ & $\sqrt{ }$ \\
\hline Dreschlera species & $\sqrt{ }$ & $N D$ & $\sqrt{ }$ \\
\hline Epicoccum species & $\sqrt{ }$ & $N D$ & $N D$ \\
\hline Fusarium oxysporium & $\sqrt{ }$ & $\sqrt{ }$ & $\sqrt{ }$ \\
\hline Mucor species & $\sqrt{ }$ & $\sqrt{ }$ & $N D$ \\
\hline Penicillium chrysogenum & $N D$ & $\sqrt{ }$ & $N D$ \\
\hline P. digitatum & $\sqrt{ }$ & $\sqrt{ }$ & $N D$ \\
\hline P. expansum & $\sqrt{ }$ & $\sqrt{ }$ & $N D$ \\
\hline P. italicum & $N D$ & $\sqrt{ }$ & $N D$ \\
\hline P. notatum & $\sqrt{ }$ & $\sqrt{ }$ & $\sqrt{ }$ \\
\hline Rhizopus stolonifer & $N D$ & $\sqrt{ }$ & $\sqrt{ }$ \\
\hline Trichoderma viride & $\sqrt{ }$ & $\sqrt{ }$ & $\sqrt{ }$ \\
\hline Yeast species & $N D$ & $N D$ & $N D$ \\
\hline
\end{tabular}

ND: not detected. 
TABLE 7. Symptoms indicated by questionnaire for employees at two libraries at the main campus of the National Research Centre (NRC), Dokki, Giza during the period of study (January to December 2013).

\begin{tabular}{|l|c|c|}
\hline \multirow{2}{*}{\multicolumn{1}{|c|}{ Symptom \% }} & Old & New \\
\cline { 2 - 3 } & 51.61 & 0.00 \\
\hline Shortness of breath & 45.16 & 12.50 \\
\hline Cough & 38.71 & 0.00 \\
\hline Wheezing & 35.48 & 8.33 \\
\hline Dry Eye & 29.03 & 4.17 \\
\hline Watering eyes & 54.84 & 16.67 \\
\hline Irritation of the eyes & 58.06 & 12.50 \\
\hline Allergic Conjunctivitis & 61.29 & 12.50 \\
\hline Sneezing & 64.52 & 4.17 \\
\hline Stuffy nose & 67.74 & 16.67 \\
\hline Coryza & 58.06 & 0.00 \\
\hline Throat irritation & 61.29 & 41.67 \\
\hline Headache & 77.42 & 45.83 \\
\hline Fatigue & 64.52 & 4.17 \\
\hline Drowsiness & 54.84 & 4.17 \\
\hline Dizziness & 35.48 & 4.17 \\
\hline Nausea & 64.52 & 4.17 \\
\hline Dermatitis & $\mathbf{3 1}$ & $\mathbf{2 4}$ \\
\hline Employees interviewed (n) & & \\
\hline
\end{tabular}

\section{Discussion}

Airborne bacteria in the present study showed variations ranged between $10^{2}$ $10^{3} \mathrm{CFU} / \mathrm{m}^{3}$ and fungi between $10^{1}-10^{2} \mathrm{CFU} / \mathrm{m}^{3}$ for indoors and outdoors concentrations. Environmental bacteria achieved the greatest microbial concentrations and mesophilic bacteria significantly differed indoors and outdoors at the old library. Generally microorganisms of indoor air depend on many factors including for example, number of people present and their hygienic standards, ventilation type and geographical location (Abdel Hameed et al., 2012). These conditions vary greatly between regions and buildings. Bacteria are mainly related to human activities, bacteria mainly shed from human skin and respiratory tracts (Fraser et al., 1977 and Chen \& Hildemann, 2009).

Higher airborne microbial concentrations indoors and outdoors were found at the old library compared to the new one. These variations are attributed to building location and ventilation type (natural ventilation). Mechanical ventilation reduces infiltration of microorganisms derived from outdoor at the new library. Moreover the old library is more impacted by traffic activities; it is faced to a busy traffic street. The age of library may also be a factor in microbial Egypt. J. Bot. 57, No.1(2017) 
generation. Older library is more likely to have issues such as dust buildup, or collected debris that could contribute to microbial generation, accumulated dust may create microenvironment for microbial growth. Moreover air movement within the libraries and outdoors help aerosolize microbial particles or keep them aloft.

Concentrations of airborne fungi inside the tested libraries were low. Indoor fungal concentrations were found in the range of $19-86 \mathrm{CFU} / \mathrm{m}^{3}$ in Poland (Karbowska-Berent et al., 2011), 524-1992 CFU/m $\mathrm{m}^{3}$ in Ethiopia (Hayleeyesus and Manaye, 2014), 29-2000 CFU/ $\mathrm{m}^{3}$ in Italy (Micheluz et al., 2015) and 59-327 $\mathrm{CFU} / \mathrm{m}^{3}$ in Cuba (Anaya et al., 2016), these results are compatible with the results in the present study. On the other hand the results in the present study were lower than those previously found in libraries Egypt. Awad and Farag (1999) found airborne bacterial and fungal concentrations at mean values of $8.9 \times 10^{3} \mathrm{CFU} / \mathrm{m}^{3}$ and $1.337 \times 10^{3} \mathrm{CFU} / \mathrm{m}^{3}$, respectively, in the National Research Center library (old library). In addition Sahab et al. (2014) found airborne bacteria and fungi concentrations in the range of 94.35-660.48 CFU x10 $10^{3} / \mathrm{m}^{3}$ and 15.72-369.45 CFU $\times 10^{3} / \mathrm{m}^{3}$ in the National Library and Archives of Egypt. These differences may be attributed to different sampling methods and culture media used for microbial growth in addition to variations in human activities and number of persons visit libraries, nowadays less number of people visit book libraries depending on website and electronic books.

There are no acceptable threshold limit values (TLVs) for bioaerosols, however some recommendations are available (Cappitelli et al., 2010). Concentrations of airborne microorganisms during the present study did not exceed the acceptable limit of $5000 \mathrm{CFU} / \mathrm{m}^{3}$ for public facilities (Górny, 2004; Augustynska et al., 2010). Moreover fungal concentrations were lower than the TLVs of the occupational exposure specified by the Polish Committee for the Highest Permissible Concentrations and Intensities of Noxious Agents at the Workplace $\left(5 \times 10^{4} \mathrm{CFU} / \mathrm{m}^{3}\right.$ for total fungi), (Skowron and Górny, 2012). Indoor fungal concentrations were $<150 \mathrm{CFU} / \mathrm{m}^{3}$ value recommended for Italian libraries (MIBAC, 2001) and the maximum value of $1000 \mathrm{CFU} / \mathrm{m}^{3}$ for archives (Nunes et al., 2013).

The variation of microbial concentrations may be attributed to differences in their size distribution. The size of microbial particles depends on many factors for example, ventilation type, location, anthropogenic activities and nature of the major source. Size of microorganism determines its behavior in the air (Nicholson, 1988). The majority of airborne bacterial concentrations were found in the coarse size fraction ( $\geq 8 \mu \mathrm{m}$ ). This is due to bacteria are mainly attached to PM, depending on the degree of urbanization, or present as aggregates, although coarse particles are settled down faster than fine ones. Bacteria are usually found in the range of 4-20 $\mu \mathrm{m}$ equivalent diameters (Noble et al., 1963). Re-suspension of dust particles increases bacterial loads in the air (Fröhlich-Nowoisky, 2011). 
However, fine fractions of fungi were found in high percentages $\geq 60 \%$, as fungal spores are found individually in the environment and/or not hydrophilic. Species of Aspergillus, Penicillium and Cladosporium with aerodynamic diameters less than $8 \mu \mathrm{m}$ were found in high percentage in air of museum store room, indicated that fungi found as single cells, spores or small fragments of mycelium (Skóra et al., 2015) It is important to sample microbial particles as size fractions, since particles with smaller diameters are more likely to enter the lungs and thus may negatively impact human health. Fine size microbial fractions are able to penetrate the respiratory system of human, causing irritation to mucous membrane of nose and eyes, inflammatory responses and allergic reactions (Kulkarni et al., 2011).

I/O ratio documents the presence/ or absence of indoor biocontaminant sources (ACGIH, 1999). In this work $\mathrm{I} / \mathrm{O}$ ratios were always $\leq 1$, suggesting no indoor bio-contaminant sources inside both libraries. I/O ratios of the environmental bacteria were relatively higher at the old library while mesophilic bacteria at the new library. This reflects role of ventilation type and number of visitors at each library respectively. A relatively high $\mathrm{I} / \mathrm{O}$ ratios of fungi (averaged 0.46) confirm derived of fungi from outdoors /or attached clothes and foot wear and settled dust.

In this work microorganism associated settled dust averaged $10^{5} \mathrm{CFU} / \mathrm{g}$ for bacteria and $10^{4} \mathrm{CFU} / \mathrm{g}$ for fungi. Microbial contents of settled dust varied depending on the amount and type of outdoor dust particles infiltrated indoors and hygienic rules. Dust can absorb moisture and provide nutrients and protection to microorganisms (Simoes et al., 2011) and it may form a microenvironment for microbial growth (Florian, 1997). Microorganisms associated dust may have potential damage of surfaces (Gallo et al., 1996) and settled dust can be dispersed elsewhere in the building under the influence of air current and human activities, constituting a source of indoor air microbial contamination (CEC, 1993). Outdoor dust particles are mainly emitted from traffic activities nearby the old library while garden activities and sweeping NRC's streets near to the new library.

Indoor and outdoor PM concentrations during the present study exceeded the limit value of $120 \mu \mathrm{g} / \mathrm{m}^{3}$ recommended by the World Health Organization (WHO, 2000) and $230 \mu \mathrm{g} / \mathrm{m}^{3}$ suggested by the Egyptian Environmental law (EEAA, 1994). However no obvious correlations were found between PM and airborne microorganisms, i.e. PM may not affect microbial concentrations. PM may serve as a carrier/ and source of nutrients for microorganisms, and at the same time may affect microbial viability and behavior (Alghamdi et al., 2014), depending on particle composition and microbial type and its tenacity to PM. PM showed positive and negative correlations with bacteria and fungi at the old and the new library. It is suggested that indoor fungi actively released indoors and dust played an important role in releasing of fungi outdoors. The negative correlations between bacteria and PM at the new library confirmed the role of PM as an essential agent for nutrient and protection of bacteria (the new library is cleaner Egypt. J. Bot. 57, No.1(2017) 
than the old one). Wan et al. (2011) found a significant correlation between bacteria and PM.

Microclimatic factors had complex influences on survival of airborne microorganisms. Relative humidity was the crucial factor supporting microbial survivability and temperature was the most detrimental factor, the influence of microclimatic factors varied depending on microbial type. Microclimatic conditions mainly cause desiccation/ or hygroscopicity effects on microorganisms (Cole and cook, 1998). Tang (2009) and Abdel Hameed et al. (2013) found different responds between bacteria and fungi to microclimatic conditions and environmental factors. Karbowska-Berent et al. (2011) found that $\mathrm{RH} \% \leq 65 \%$ detrimentally affected bacterial growth, and $\mathrm{RH} \%$ had significant influence on fungal concentrations, while in other cases $\mathrm{T}^{\circ} \mathrm{C}$ and $\mathrm{RH} \%$ had no significant influence on microbial populations.

Gram positive cocci and bacilli were quite numerous in the air state and settled dust. These organisms normally exist on human skin and related to human activities. Gram positive bacteria are more resistant to sever environmental conditions (Beggs, 2003). Staphylococcus bacteria are related to human skin and Bacillus and spore forming bacteria are numerous in dust particles and their concentrations differed from place to place depending on microenvironment and human activities (Burkowska et al., 2012). It has been reported that high concentration of Bacillus species in indoor air is usually indicative of water damage/ or lack of building maintenance (Baxter et al., 1981). Bacillus bacteria have ability to form spores, allow them to resist the unfavorable conditions long period of time (Karbowska-Berent et al., 2011). Bacillus and Streptomyces species constitute potential risk for paper, because they can produce cellulose degrading enzyme (Ramirez and Coha, 2003).

The results in the present study agree with the findings obtained by other investigators. Borrego and Perdomo (2012) found that Gram positive cocci and bacilli bacteria are abundant in libraries in Cuba. Moreover, species of Micrococcus (Harkawy et al., 2011), Staphylococcus (Hayleeyesus and Manaye, 2014) and Bacillus (Skóra et al., 2015) were found to be the common bacterial genera in indoors environment of libraries and archives.

The majority of fungal taxa isolated from libraries are commonly existed in the ambient air such as Aspergillus, Penicillium, and Cladosporium. Aspergillus and Penicillium are able to colonize different substrates and resist unfavorable conditions (Araujo and Cabral, 2010). Cladosporium, Penicillium and Aspergillus were the common indoor fungi in libraries and archives (Micheluz et al., 2015; Skóra et al., 2015 and Anaya et al., 2016). A. versicolor, Paecilomyces and A. fumigatus were numerous in the library in Poland (Cieplik, 1997). Penicillium, A. flavus, Trichoderma viride, Alternaria tenuis and A. niger constituted $54.42 \%$ of the total air spores in the National Library of Egypt (Sahab et al., 2014). 
The identification of fungi associated settled dust (Fig. 5b) showed that composition of fungal taxa reflected composition of airborne fungi (Fig. 5a), confirming that settled dust is an important source of airborne fungi. Nigrospora species and Trichoderma viride were only found in the settled dust whereas Monilia and Mucor in the air state. Cladosporium, Penicillium dominated in the air state, and yeast in the settled dust. This may be attributed to periodicity of source, location and, and microclimatic conditions. Moreover aerodynamic diameter determines fungal transport in the air, and different fungal size distribution would be expected to cause different behavior in the air (Baron and Willeke, 1993). Pasanen et al. (1991) reported that the minimum air velocity at which Cladosporium released spores was $1 \mathrm{~m} / \mathrm{s}$ and $0.5 \mathrm{~m} / \mathrm{s}$ for release of Aspergillus and Penicillium, respectively.

The qualitative composition of air microorganisms is important indoor issue due to the fact that they may pose threats to book collections and human health. Some fungi can cause allergy-mediated diseases (Karbowska-Bernet et al., 2007) and mycotoxicosis in human (Flanningan, 2001). Aspergillus, Mucor and Rhizopus pose threat to vulnerable individuals (Soleimani et al., 2013). Aspergillus fumigatus, A. flavus and Stachybotrys chartarum should not be present in the air of library and their concentrations should be zero CFU $/ \mathrm{m}^{3}$ (Górny et al., 2004 and Augustynska et al., 2010). Staphylococcus and Bacillus bacteria were numerous in the environment and may have been transferred to book as a result of contact with readers causing respiratory diseases.

In this work, many of fungal isolates had enzymatic properties; A. flavus, Curvulari apallescens, Fusarium oxysporium, Penicillium notatum and Trichoderma viride had proteolytic, lipolytic and cellulolytic activities, which may damage library materials. May et al. (1993) listed fungi among the major agents of microbial deteriorations of surfaces and Leznicka et al. (1988) pointed to the significance of dematiaceous fungi in the staining and deterioration of works of arts. Urzi et al. (2001) reported that once fungi settle on the colonize surface they attribute a great variety of alternation like black paints, intergranular growth and biopitting. Fungal species exhibit strong cellulolytic (e.g. Trichoderma, Penicillium, Botrytis, Chaetomium, Stemphylium and Alternaria), proteolytic (e.g. Mucor, Aureobasidium, Chaetomium, Trichoderma, Verticillium and Epicoccum) and lipolytic (as above plus Paecilomyces) properties (Karbowska-Berent et al., 2011).

Although the obtained viable microbial concentrations were low in the air or settled dust, they may pose health threats. Indoor microorganisms may cause health symptoms (Mishra et al., 1992). In the present work the severity of health symptoms was higher among employees at the older library, indoor air quality was worse at the older library. The development of specific symptoms (e.g., multiple respiratory symptoms and atopic group) may not obviously clear among employees because several symptoms may be related to indoor environmental factors including PM, bio-contamination, ventilation, Job stress, space and heat comfort (Mendell et al., 1996). Generally, there is a lack of information of how Egypt. J. Bot. 57, No.1(2017) 
the different physical, psychological stress, chemical and biological contaminants interacts to produce discomfort and complaints in non-industrial settings in Egypt. The limitation in the interview is the employee cannot accurately determine that the health symptoms may be building related.

\section{Conclusion}

Little information is available on microbiological air quality in nonindustrial settings in Egypt. This work aims to evaluate microbial indoor air quality at libraries and factors affecting their prevalence. The concentrations and types of microorganisms were library dependent, the older library had higher airborne microbial concentrations than the new one. Dust buildup and ventilation were considered to be the main factors influence indoor microbial concentrations. Environmental bacteria achieved the greatest airborne microbial concentrations while mesophilic bacteria significantly differed between indoors and outdoors at the older library. I/O ratios of airborne microorganisms were always $\leq 1$ confirming no permanent indoor bio-contaminant sources at both libraries. Microclimatic factors had complex influences on airborne microbial survivability; however relative humidity was the crucial factor supporting their survival, depending on microbial type. Gram positive cocci and bacilli were commonly found in the air state and settled the dust. Aspergillus, Penicillium and Cladosporium were the common fungal species. A composition of fungi taxa associated settled dust reflected the composition of airborne fungi. Many of the isolated fungal taxa had enzymatic properties which may damage library materials. Although the microbial concentrations were low but the obtained microorganisms might pose health threats.

Acknowledgment This work was supported by National Research Centre (NRC) and The Academy of Scientific Research \& Technology (ASRT).

\section{References}

Abdel Hameed, A.A., Khoder, M.I., Ibrahim,Y.H., Saeed, Y., Osman, M.E. and Ghanem, S. (2012) Study on some factors affecting survivability of airborne fungi. Science of the Total Environment 414, 696-700.

Abdel Hameed, A.A., Shawn, G.G., Patrick, T.M. and Christopher, F.G. (2013). Coarse and fine culturable fungal air concentrations in urban and rural homes in Egypt. International Journal of Environmental Research and Public Health 10(3), 936-949.

ACGIH (American conference of governmental industrial hygienists), (1999) Bioaerosols: assessment and control. American conference of governmental industrial hygienists, Cincinnati, Ohio.

Alghamdi, M.A., Shamy, M., Redal, M.A., Khder, M., Abdel Hameed, A. and Elserougy, S. (2014) Microorganisms associated particulate matter: A preliminary study. Science of the Total Environment 479-480, 109-16.

Egypt. J. Bot. 57, No.1(2017) 
Ammar, M.S., Louboudy, S.S. and Abdul- Raouf, U.M. (1991) Distribution, total viable bacteria and identification of the most potent proteolytic bacterial strains isolated from Aswan City. Az. J. Microbiol. 11, 224-238.

Anaya, M., Borrego, S.F., Gamez, E., Castro, M., Molina, A. and Valdes, O. (2016) Viable fungi in the air of indoor environments of the National Archive of the Republic of Cuba. Aerobiologia 32 (3), 513-527.

Andersen, A.A. (1958) New sampler for the collection, sizing, and enumeration of viable airborne particles. Journal of Bacteriology 6, 471-484.

Araujo, R., Cabral, J.P. (2010) Fungal Air Quality in Medical Protected Environments. (Chapter 17). In Air Quality by Ashok K, ISBN 978-953-307-131-2.

Augustynska, D., Posniak, M. and Miedzyresortowakomisja, ds. (2010) Najwyzszychdopuszczalnychstezeninatezenczynnikówszkodliwychdlazdrowiawsrodo wiskupracy - czynnikiszkodliwe w _srodowiskupracy - warto_scidopuszczalne. Warsaw: CIOP-PIB.

Awad, A.A. and Farag, S.A. (1999) An indoor bio-contaminants air quality. International Journal of Environmental Health Research 9,313 -319.

Barnett, H.L. and Hunter, B.B. (1999) Illustrated genera of imperfect fungi 4th edition. St. Paul, MN: The American phytopathological society, APS, p. 218.

Barnett, H.L. (1972) Illustrated Genera of Imperfect Fungi. Burgess Publishing Company, Minneapolis, Minnesota.

Baron, P.A., Willeke, K.. Willeke, K. and Baron, P.A. (1993) "Aerosol Measurement: Principles, Techniques And Applications. New York: Van Nostrand Reinhol. pp. 822.

Baxter, C.S., Wey, H.E. and Burg, W.E. (1981) Prospective analysis of the potential risk associated with inhalation of aflatoxin-contaminated grain dusts. Food Cosmetics Toxicology 19, 763-769.

Beggs, C.B. (2003) The airborne transmission of infection in hospital buildings: fact of fiction? Indoor and built Environment. 12, 9-18.

Berent, J.K., Górny, R.L., Strzelczyk, A.B. and Wlazlo, A. (2011) Airborne dust borne microorganisms in selected polish libraries and archives. Building and Environment 46 (10), 1872-1879.

Bland, S., Douglas, E.E. (1977) Semi quantitative plate assay for determination of cellulase production by Trichoderma viride. Applied and Environmental Microbiology 33(1), 178-83.

Borrego, S. and Perdomo, I. (2012) Aerobiological investigations inside repositories of the National Archive of the Republic of Cuba. Aerobiologia 28, 303-316. 
Borrego, S., Guiamet, P., Gomez, S., Batistini, P., Garcia, M., Lavin, P. and Perdomo, I. (2010) The quality of air at archives and the biodeterioration of photographs. International Biodeterioration \& Biodegradation 64, 139-145.

Burkowska, A. and Kalwasińska, A. et al. (2012) Airborne mesophilic bacteria at the Ciechocinek health resort. Polish Journal of Environmental Studies 21(2), 307-312.

Cappitelli, F., Pasquariello, G,, Tarsitani,G., Sorlini, C. and Scriptamanent (2010) Assessing microbial risk to paper heritage. Trends in Microbiology 18, 538-542.

CEC (1993) Report No. 12. Biological particles in indoor environments, Commission of the European Communities, Luxembourg 1994.

Chen, Q. and Hildemann, L.M. (2009) The effects of human activities on exposure to particulate matter and bioaerosols in residential homes. Environmental Science and Technology 43, 4641-4646.

Cieplik, Z. (1997) Zagrzybienie ksie,gozbioru Biblioteki Polonistycznej wydzialu Filologicznego Uniwersytetu_Sla_skiego.SStud Bibliol 10,107-19.

Ciferri, O., Becker, M.E. and De Rossi, E. (2003) Characterization of bacteria isolated from naturally aged silk fi broin. In: "Art, Biology, and Conservation: Biodeterioration of Works of Art". Koestler RJ, Koestler VH, Charola AE, NietoFernandez FE (Eds.). 190-207. Yale University Press, New York.

Cole, E.C. and Cook, C.E. (1998) Characterization of infectious aerosols in health care facilities: an aid to effective engineering controls and preventive strategies. American Journal of infection control 26(4), 453-464.

Cowan, S.T. (1974) "Cowan and Steel's Manual for the Identification of Medical Bacteria”. $2^{\text {nd }}$ ed., 193-227. Cambridge University Press, England.

Crandell, M.S., Karl Sieben, W. and Malkin, R. (1996) HVAC and building environmental findings and health symptoms associations in 80 office buildings. N IAQ961 paths to better building environments health symptoms in building occupants, Baltimore, Maryland, USA. October 6-8; 1-3-108.

Eduard, W. (2009) Fungal spores: A critical review of the toxicological and epidemiological evidence as a basis for occupational exposure limit setting. Critical Reviews in Toxicology, 39(10),799-864.

EEAA (1994) 'Egyptian Environmental Affair Agency". Environmental protection law No. 4.

Ellis, M.B. (1971) "Dematiaceous Hyphomycetes". p. 608. The Western Press Ltd: London and Readin common wealth Mycological Institute Kew, Surrey, UK.

Flanningan, B. (2001) Microbial aerosols in buildings: origins, health implications and controls. p. 11-27. In: II konferencja naukowa "Rozk1ad i Korozja Mikrobiologiczna Materialów Technicznych", qód_z. 
Florian, M.L. (1997) "Heritage Eaters". pp. 1-12. Insects and Fungi in Heritage Collections. James and James Science Publishers Ltd., London.

Fraser, D.W., Tsai, T.R., Overstein, W., Parkin, W.E., Becham, H. and Shanar, P.G. (1977) Legionnaries disease description of an epidemic of pneumonia. The New England Journal of Medicine 297,1189-1197.

Fröhlich-Nowoisky, J., Burrows, S.M., Xie, Z., Engling, G., Solomon, P.A. and Fraser, M.P. et al (2011) Biogeography in the air: fungal diversity over land and oceans. Biogeosciences Discussions 8,7071-96.

Gallo, F. (1993) Aerobiogical research and problems in libraries. Aerobiologia 9(2-3), 117-130.

Gallo, F., Valenti, P., Colaizzi, P., Sclocchi, M.C., Pasquariello, G., Scorrano, M., Maggi, O. and Persiani, A.M. (1996) Research on the viability of fungal spores in relation to different microclimates and materials. International Conference on Conservation and Restoration of Archive and Library Materials, vol. I. Erice, Italy, 22-29 Avril 1996; Roma, Italy, pp. 177-193.

Górny, R.L. (2004) Fungal and bacterial propagules as indoor air contaminants: characteristics, release mechanisms, detection. Sosnowiec: Institute of Occupational Medicine and Environmental Health Publishing.

Harkawy, A., Górny, R.L., Ogierman, L., Wlazło, A., Lawniczek-Wałczyk, A. and Niesler,A. (2011) Bioaerosol assessment in naturally ventilated historical library building with restricted personnel access. Annals of Agricultural and Environmental Medicine 18(2), 323-329.

Hayleeyesus, S. and Manaye, A. (2014) Microbiological Quality of Indoor Air in University Libraries. Asian Pacific Journal of Tropical Biomedicine 4(1), S312-S317.

Hussein, N.A. (2002) Studies on the genus Aspergillus and its teleomorphs in Egypt. PhD Thesis, Botany department, Faculty of science, Assiut University, Assiut, Egypt.

Karbowska-Berent, J., Jarosinska, D. and Muszynska-Graca, M. (2007) Ocena stopnia narazenia pracowników bibliotek i archiwów na alergie i grzybice w _srodowiskupracy. Notes Konserwat 11, 255-66.

Karbowska-Berent, J., Górny, R.L., Strzelczyk, A.B. and Wlazio, A. (2011) Airborne and dust borne microorganisms in selected Polish libraries and archives. Building and Environment 46, 1872-1879.

Kulkarni, P., Baron, P.A. and Willeke, K. (2011) "Aerosol Measurement: Principles, Techniques, and Applications". New York: Wiley.

Leznicka, S., Strzelczyk, A. and Wandtychowska, D. (1988) Removing of fungal stains from stone-works. In: IV International Congress on Deterioration and Conservation of Stone, Vol. 2, Nicolaus Copernicus University, Torun, pp. 102-110. 
Maggi, O., Parsiani, A.M., Gallo, F., Valenti, P., Pasquariello, G. and Sclocchi, M.C. (2000) Airborne fungal spores in dust present in archives: proposal for a detection method, new for archival materials. Aerobiologia 16, 429-34.

May, E., Lewis, F.J., Pereira, S., Tayler, S., Seaward, M.R.D. and Allsopp, D. (1993) Microbial deterioration of building stone - a review. Biodeterioration Abstracts 7,109123.

Mendell, M.J., Sieber, W.K., Dong, M.X., Malkin, R. and Wilcox, T. (1996) "Symptom prevalence distributions in US office buildings investigated by NIOSH for indoor environmental quality complaints". Presented at The Seventh International Conference on IAQ and Climate, Nagoya, Japan.

Mesquita, N., Portugal, A., Videira, S., Rodrıguez-Echeverrı, S., Bandeira, A.M.L. and Santos, M.J.A. (2009) Fungal diversity in ancient documents. A case study on Archive of the University of Coimbra. International Biodeterioration and Biodegradation 63, 626-629.

MIBAC (2001) Attod'indirizzosuicriteritecnico-scientifici e Sugli Standard di Funzionamento Esviluppodeimusei, D.Lgs n.112/98 art.150 comma 6.

Micheluz, A., Manente, S., Tigini, V., Prigione, V., Pinzari, F., Ravagnan, G. and Varese, G. (2015) The extreme environment of a library: Xerophilic fungi inhabiting indoor niches. International Biodeterioration and Biodegradation 99: 1-7.

Mishra, S.K., Ajello, L., Ahearn, D. G. (1992) Environmental mycology and its importance to public health. Journal of Medical and Veterinary Mycology 30 (1), 287305.

Mouli, C.P., Mohan, S.V. and Reddy, S. (2005) Assessment of microbial (bacteria) concentrations of ambient air at semi- arid urban region. Influence of meteorological factors. Applied ecology and environmental research 3 (2), 139-149.

Nicholson, K.W. (1988) The dry deposition of small particles: a review of experimental measurements. Atmospheric Environment 22, 2653 -2666.

Noble, E.C., Lidwell, O.M. and Kingston, D. (1963) The size distribution of airborne particles carrying micro-organisms. Journal of Hygiene (Cambridge) 61, 385-391.

Nunes, I., Mesquita, N., Cabo Verde, S., Leit aoBandeira, A.M., Carolino, M.M., Portugal, A. and Botelho, M.L. (2013) Characterization of an airborne microbial community: a case study in the archive of the University of Coimbra, Portugal. International Biodeterioration and Biodegradation 79, 36-41.

Osman, M.E., Om Kalthoum, H.K. and El-Shaphy, A.A. (2008) Production of cellulase and pectinase from some aquatic hyphomycetes. Research journal of microbiology $\mathbf{3}$, 213-224.

Pasanen, A.L., Pasanen, P., Jantunen, M.J. and Kalliokoski, P. (1991) Significance of air humidity and air velocity for fungal spore release into the air. Atmospheric Environment 25, 459-462. 
Pitt, I.J. and Hocking, A. (2009) Fungi and food spoilage "Springer Science and Business Media”, LLC, 233 Spring street, New York, NY 10013, USA.

Ramırez, P. and Coha, J. M. (2003) Enzymatic degradation of cellulose for thermophilic actinomycete: Isolation, characterization and cellulolytic activity determination. Revista Peruana de Biologia 10, 67-77.

Raper, K.B. and Fennell, D.I. (1977) The genus Aspergillus. R.E. Krieger Publishing Company, Huntington, NY, USA.

Ritchkoff, A., Viitanen, H. and Koskela, K. (2000) The response of building materials to the mould exposure at different humidity and temperature conditions. Proceedings of healthy buildings 3, 317-322.

Sahab, A.F., Sidkey, N.M., Abed, N. N. and Mounir, A. (2014) Studies on Indoor Air Quality in the Repositories of the National Library and Archives of Egypt. International Journal of Science and Research (IJSR) 3(11).

Sarica, S.Ö., Asan, A., Tungan,Y. and Ture, M. (2005) Airborne fungal concentrations in east patch of Edirne city (Turkey) in autumn using two sampling methods. Trakya university journal of scientific research 6 (1), 97-106.

Simoes, S., Almeida, A., Junior and Hohn, R. (2011) Fungal microbiota in air conditioning installed in both central western region. Mat Grosse, Brazil. Mycopathologia 172, 109-116.

Singh, K., Frisvad, J.C., Thrane, U. and Mathur, S.B. (1991) An illustrated manual on identification of some seed-borne Aspergilli, Fusaria, Penicillia and their mycotoxins. Danish Government Institute of Seed Pathology for Developing Countries. RyvangsAlle' 78 DK-2990 Hellerup: Denmark.

Singh, R. N., Gupta, V. K., Goswami and Gupta, R. (2006) A simple activity staining protocol for lipases and esterases. Applied Microbiology and Biotechnology 70, 679682.

Skóra, J., Beata, G. and Katarzyna, P. (2015) Assessment of microbiological contamination in the work environments of museums, archives and libraries. Aerobiologia 31, 389-401.

Skóra, J., Zduniak, K., Gutarowska, B. and Rembisz, D. (2012) Harmful biological agents at museum workposts. Medycyna Pracy 63(2), 153-165. Polish.

Skowron, J. and Górny, R. (2012) Harmful biological agents. In: D. Augustynska, \& M. Posniak, (Eds.), The Interdepartmental Commission for Maximum Admissible Concentrations and Intensities for Agents Harmful to Health in the Working Environment: limit values Warszawa: Centrally Instytut Ochrony Pracy-Pan'stwowy Instytut Badawczy. Polish.

Soleimani, Z., Goudarzi, G., Naddafi, K., Sadeghinejad, B., Latifi, S.M. and Parhizgari, N. (2013) Determination of culturable indoor airborne fungi during normal and dust event days in Ahvaz, Iran. Aerobiologia 29, 279-90.

Egypt. J. Bot. 57, No.1(2017) 
Tang, W. (2009) The effect of environmental parameters on the survival of airborne infectious agents. Journal of the Royal society interface 6(6), S737-S746.

Tao, Y., Zhou, M.S. and Xie, S. (2014) Air pollution and hospital admission for respiratory diseases in Lanzhou, China. Environmental Pollution 185, 196- 201.

Thomas, R.J. (2013) Particle size and pathogenicity in the respiratory tract. Virulence 15:4(8), 847-858.

Urzi, C. and Realini, M. (1998) Colour changes of Noto's calcareous sandstone as related to its colonization by microorganisms. International Biodeterioration and Biodegradation 42, 45-54.

Urzi, C., de Leo, F., Salamone, P. and Criseo, G. (2001) Airborne fungal spores colonizing marbles exposed in the terrace of Messina Museum, Sicily. Aerobiologia 17, 11-17.

Wan, G.W., Chung, F.F. and Tang, C.S. (2011) Long-Term Surveillance of Air Quality in Medical Center Operating Rooms. American Journal of Infection Control 39, 302308.

WHO (World Health organization), (2000) “Guidelines For air Quality”. Geneva.

Wiszniewska, M., Walusiak-Skorupa, J., Pannenko, I., Draniak, M. and Pałczynski, C. (2009) Occupational exposure and sensitization to fungi among museum workers. Occupational Medicine, 59, 237-242.

(Received 13/10/2016; accepted $14 / 12 / 2016)$ 


\section{تلوث الهواء الميكروبي و العوامل التى تؤثر في حدوثه في بعض

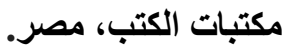

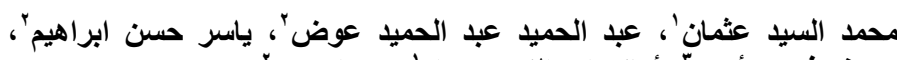

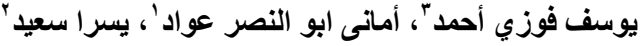

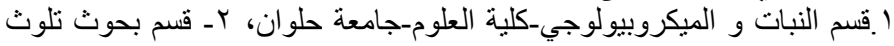

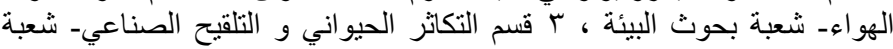 البحوث البيطريةـ المركز القومي للبحوثـ مصر.}

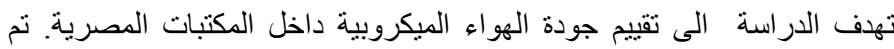

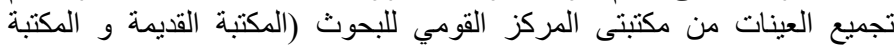

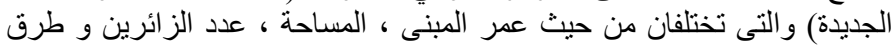

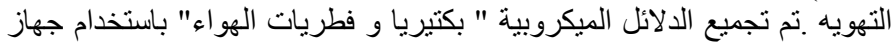

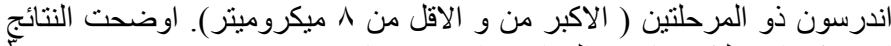

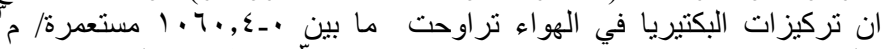

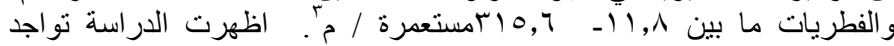

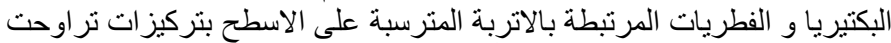

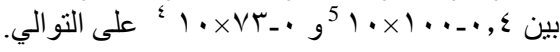

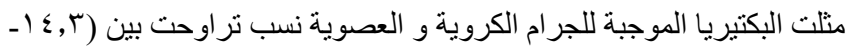

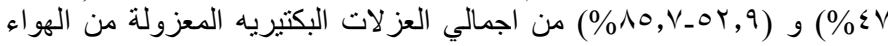

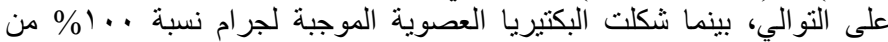

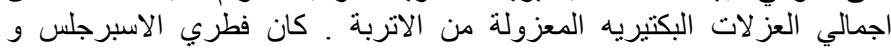

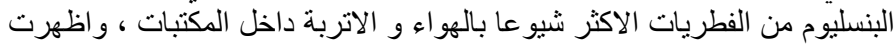

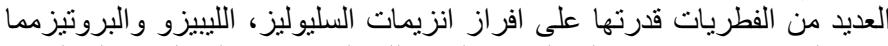

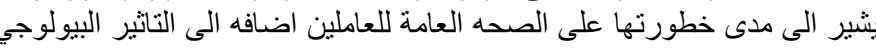

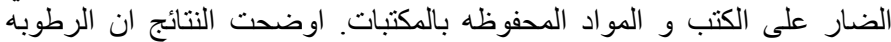
النسبية من العو امل الاساسية التى تؤثر على حيوية الكائنات الحية بالبيئة الداخلية النية النية ل اللمكتبات. 